\title{
BMJ
}

\section{Renal screening in children after exposure to low dose melamine in Hong Kong: cross sectional study}

\author{
Hugh S Lam, assistant professor, ${ }^{1}$ Pak C Ng, chairman and professor of paediatrics, ${ }^{1}$ Winnie C W Chu,

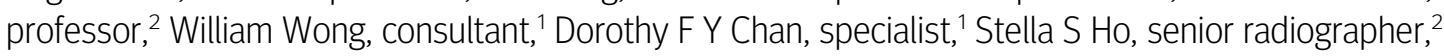 \\ Ka T Wong, consultant, ${ }^{2}$ Anil T Ahuja, chief of service, ${ }^{2}$ Chi K Li, chief of service ${ }^{1}$
}

${ }^{1}$ Department of Paediatrics, The Chinese University of Hong Kong

${ }^{2}$ Department of Diagnostic Radiology and Organ Imaging, The Chinese University of Hong Kong Correspondence to: H S Lam, 6/F Clinical Sciences Building, Department of Paediatrics,

Prince of Wales Hospital, Sha Tin, New Territories, Hong Kong hshslam@cuhk.edu.hk

Cite this as: $B M J$ 2008;337:a2991 doi:10.1136/bmj.a2991

\section{ABSTRACT}

Objective To investigate the renal outcomes of children after exposure to low dose melamine in Hong Kong. Design Cross sectional study.

Setting Special assessment centres, Hong Kong. Participants 3170 children (1422 girls and 1748 boys) aged 12 years or less referred from territory-wide primary care clinics after daily consumption for one month or more of milk products tainted with melamine.

Main outcome measures Presence of renal stones and haematuria.
Results One child had a confirmed renal stone, seven were suspected of having melamine related renal deposits, and $208(6.6 \%)$ were positive for blood in urine by reagent strip. A proportion of these children were followed up at the special assessment centre, but only $7.4 \%$ of those positive for blood on reagent strip were confirmed by microscopy, suggesting an overall estimated prevalence of less than $1 \%$ for microscopic haematuria.

Conclusions No severe adverse renal outcomes, such as acute renal failure or urinary tract obstruction, were detected in children after exposure to low dose melamine. Our results were similar to territory-wide findings in Hong Kong. Even including the seven children with suspected renal deposits, the prevalence of suspected melamine related abnormalities on ultrasonography was only $0.2 \%$. None of these children required specific treatment. The prevalence of microscopic haematuria was probably overestimated by the reagent strip. These data suggest that large scale and urgent screening programmes may not be informative or cost effective for populations who have been exposed to low dose melamine.

\section{INTRODUCTION}

Since the first media reports on 11 September 2008 linking an outbreak of renal diseases among children on the mainland of China to consumption of milk products contaminated with melamine, an estimated 50000 or more children have been affected. ${ }^{12}$ More than 100 became seriously ill and at least four deaths have been attributed to melamine. ${ }^{3}$

Melamine is a triazine compound and is an essential component of materials such as flame retardants, glues, and plastics. ${ }^{45}$ In the mainland, addition of this compound to milk intended for human consumption allowed diluted milk to appear to contain satisfactory amounts of protein and to pass food quality tests. Little is known about the adverse effects of melamine consumption in humans. The main adverse effects shown in studies on animals were kidney related, including renal calculi, renal tubular necrosis, melamine crystalluria, and haematuria. ${ }^{46}$ On the mainland where young children were exposed to high doses of melamine, adverse renal effects, including renal stones and acute renal failure, have been reported. ${ }^{4}$ Although milk products tainted with melamine were found to be commercially available outside the mainland-for example, the Hong Kong Special Administrative Region, these exported products had much lower concentrations of melamine. Melamine contaminated milk products available on the mainland could contain as much as $2563 \mathrm{mg} / \mathrm{kg}$ of melamine, whereas the most severely contaminated milk product manufactured on the mainland but commercially available in Hong Kong contained $68 \mathrm{mg} / \mathrm{kg}^{8}{ }^{8}$

In view of the wide range of food products found to be contaminated with melamine and the proximity of Hong Kong to the mainland, parents throughout the territory were anxious about the possible adverse health effects on their children of consuming tainted milk products. In the absence of good evidence to determine the risk of melamine related complications in children in Hong Kong, the government initiated a territory-wide screening programme for renal complications in children aged 12 years or less who had consumed the contaminated milk products. In view of the lack of published data on the consequences of exposure to low dose melamine - that is, doses below the tolerable daily intake recommended by the US Food and Drug Administration of $0.63 \mathrm{mg} / \mathrm{kg} / \mathrm{day},{ }^{4}$ we report the short term findings from our cohort.

\section{METHODS}

General outpatient clinics at primary care level throughout Hong Kong were designated as first level screening centres. Hong Kong residents could walk in for assessment without appointment. Children who were aged 12 years or less and had consumed 
melamine tainted milk products daily for one month or more were referred to special assessment centres for renal assessments. We defined melamine tainted milk products as those known to be contaminated with melamine according to test results posted by the Hong Kong government. ${ }^{8}$ We recorded the brands of contaminated products, the daily intake, and the duration of continuous consumption. In view of the large degree of uncertainty and rapidly expanding list of confirmed tainted products during the early weeks of screening, the Hong Kong government refrained from setting minimum consumption volumes or estimated daily intake thresholds for melamine below which the child would not be referred for renal assessment.

All children at our special assessment centre underwent renal ultrasonography and urinalysis. Ultrasound examinations were done by experienced sonographers and radiologists. Positive findings were cross checked by the chief consultant radiologist before being reported. Although the sonographers and radiologists were not purposely blinded to history of exposure, this information was not available to those who carried out the renal ultrasonography. Furthermore, with the large numbers of children for each scanning session, the sonographers or radiologists lacked the time to find out about consumption or to estimate the level of exposure to melamine. In practice therefore ultrasonography was essentially done blinded to exposure history.

Urine was analysed using Combur-Test strips (Roche Diagnostics, Mannheim Germany). In view of the large number of children screened daily, those with urine positive for blood on reagent strip testing were referred back to the local primary care clinics for further investigation and follow-up if no abnormalities were found on ultrasonography or for renal function. Consequently, with smaller numbers of children seen, urine microscopy was done on all those with urine positive for blood on reagent strips to further assess the haematuria. In addition, blood samples were taken for assessment of urea and creatinine levels in all but one of the children who had abnormalities on ultrasonography or urinalysis.

\section{RESULTS}

Between 25 September and 30 October 2008 the special assessment centre at the Prince of Wales Hospital assessed 3170 children (1422 girls and 1748 boys), mean age 6.4 (range 0.1 to 12.9 ) years, who had consumed milk products tainted with melamine. Milk intake had been on a regular basis for at least one month, from twice a week to daily. The amount of milk consumed was variable, with self reported daily volumes ranging from $250 \mathrm{ml}$ to more than 1.5 litres. All reported regularly consuming at least one of the milk products that had been found to contain melamine. ${ }^{8}$ The estimated melamine intake of the eight children with renal stones or deposits was between $0.01 \mathrm{mg} / \mathrm{kg} /$ day and $0.21 \mathrm{mg} / \mathrm{kg} / \mathrm{day}$, which did not exceed the tolerable daily intake of $0.63 \mathrm{mg} / \mathrm{kg} /$ day. ${ }^{4}$
A renal calculus $(7 \mathrm{~mm})$ without associated obstructive hydronephrosis was found in one child $(0.03 \%)$. Seven children $(0.2 \%)$ had small hyperechoic renal foci $(<4 \mathrm{~mm})$ at or close to the renal papillae. They all had a history of good health and only one child with small hyperechoic renal foci had urinary symptoms (dysuria and urinary frequency). The urinary calcium to creatinine ratio was normal in all children with renal stones or deposits. Incidental findings were identified in 17 children $(0.5 \%)$, which were considered unrelated to melamine intake (table).

On reagent strip urinalysis, 115 girls $(8.0 \%)$ and 93 boys $(5.3 \%)$ were positive for blood, 34 girls $(2.4 \%)$ and 25 boys $(1.4 \%)$ were positive for protein, and 106 girls $(7.6 \%)$ and two boys $(0.1 \%)$ were positive for leucocytes. Of the 54 children with positive results for blood between 15 and 29 October, red blood cells in the urine could be confirmed by microscopy in only four. This would reduce the overall estimated prevalence of haematuria to less than $1 \%$. One 3 year old boy and one 5 year old girl had low calculated creatinine clearance (54 $\mathrm{ml} / \mathrm{min} / 1.73 \mathrm{~m}^{2}$ and $59 \mathrm{ml} / \mathrm{min} / 1.73 \mathrm{~m}^{2}$, respectively $)^{9}$; however, no other abnormalities were found on further assessment. Only one of the eight children with suspected renal stones or deposits showed any abnormalities on urinalysis (blood ++++ by reagent strip), and all had normal plasma creatinine concentrations.

\section{DISCUSSION}

In the absence of any relevant data on acute outcomes in children exposed to low dose melamine during the months before September 2008 in China, the government of the Hong Kong Special Administrative Region reacted promptly by screening large numbers of children for renal complications. Unlike children living on the mainland of China, who were exposed to much higher doses of melamine than children in Hong Kong, no serious adverse renal outcomes were detected in our screening programme. We speculate that the disparity in outcomes between children who were assessed in

Results of renal ultrasonography and urinalysis in $\mathbf{3 1 7 0}$ Chinese children who consumed melamine tainted milk products between 25 September and 30 October 2008

\begin{tabular}{lcc} 
Abnormality & $\begin{array}{c}\text { No with } \\
\text { abnormality }\end{array}$ & Prevalence (\%) \\
Renal calculus & 1 & 0.03 \\
\hline Renal deposit & 7 & 0.22 \\
\hline Other renal abnormalities* & 17 & 0.54 \\
\hline Haematuria & 208 & 6.56 \\
\hline Proteinuria & 59 & 1.86 \\
\hline Leucocytes & 108 & 3.40 \\
\hline $\begin{array}{l}\text { Other abnormalities on } \\
\text { urinalysis† }\end{array}$ & 5 & 0.16
\end{tabular}

*Pelviectasia $(n=4)$, tiny milk of calcium cysts $(n=3)$, renal agenesis $(n=2)$, polycystic renal disease $(n=2)$, horseshoe kidney $(n=1)$, transient dilation of ureter $(n=1)$, ureterocele $(n=1)$, small scarred kidney $(n=1)$, ovarian dermoid $(n=1)$, and ovarian cystadenoma $(n=1)$.

tOther abnormalities on urinalysis: ketones $(n=1)$, bilirubin $(n=1)$, nitrites $(n=1)$, and glucose $(n=4)$. 


\section{WHAT IS ALREADY KNOWN ON THIS TOPIC}

Exposure to high dose melamine was linked to an outbreak of severe renal diseases among children in China

Little is known about the renal outcomes of children after exposure to low dose melamine

\section{WHAT THIS STUDY ADDS}

Large numbers of children in Hong Kong were screened for acute renal complications within a short period

No severe adverse renal outcomes were detected

Urgent and large scale renal screening in children with a history of exposure to low dose melamine may not be necessary

Hong Kong and those living on the mainland was the large difference in concentrations of melamine in the milk products consumed.

\section{Comparison with other studies}

The pattern of complications detected in the territorywide screening in the Hong Kong Special Administrative Region was similar to that of our cohort. Of 17667 children screened at all special assessment centres up to 5 November 2008, only $10(0.06 \%)$ had renal stones $(\geq 4 \mathrm{~mm})$ detected. Echogenic foci closely related to the renal papillae are unusual findings on ultrasonography in asymptomatic children. In a report on 196 cases of microlithiasis in children, all but 13 presented with abdominal or genitourinary symptoms. ${ }^{10}$ A large proportion also had urinary abnormalities, including haematuria $(61 \%)$ and hypercalcuria $(38 \%)$. In our children with renal deposits, only one had haematuria, but all had a normal urinary calcium:creatinine ratio. These hyperechoic lesions were noted to have less acoustic shadowing than usual renal stones. The features of these suspected melamine related renal deposits on ultrasonography resemble gallbladder sludge rather than calcified stones. ${ }^{11}$ Even including these seven children with possible renal deposits, only a small percentage $(0.2 \%)$ of children exposed to relatively low dose melamine were affected. None of our children developed acute renal failure or urinary tract obstruction, or required treatment. However, the high prevalence of abnormalities in urine is unusual. It is possible that a large proportion was false positive, as suggested by the small number of children with red blood cells in urine confirmed by microscopy. As reagent strips can be more sensitive than microscopy, ${ }^{12}$ however, some of these children may genuinely have had mild haematuria. Results from a screening programme in Japan showed that in 6197 school aged children, dipstick alone detected occult blood in $4.1 \%$ and protein in $2.1 \%{ }^{13}$ The proportion of children with haematuria detected by reagent strip testing was slightly higher in our cohort $(6.6 \%)$. Using a multilevel screening algorithm in a larger cohort (23 121 Japanese children), haematuria was detected in $0.7 \%$ and proteinuria in less than $0.01 \% .^{14}$ The proportion of children with haematuria confirmed by microscopy in Hong Kong is similar to that in Japan.

\section{Strengths and limitations of study}

The catchment area of our centre includes the northern areas of Hong Kong closest to the border with the mainland. Our cohort therefore includes a large mobile population that travels often and regularly between the Hong Kong Special Administrative Region and the mainland. A large population of the children are born in Hong Kong but live across the border. Children seen at our centre would therefore be expected to be at highest risk of exposure to melamine tainted milk products within Hong Kong, but probably of lower risk compared with infants and children living on the mainland. To date our centre has screened the largest number of children in Hong Kong.

A limitation of our analyses is the absence of a reliable biomarker of melamine exposure. In view of the short plasma half life of melamine (three hours), blood concentrations are unreliable. ${ }^{4}$ The concentration of melamine in urine is also an unhelpful marker as levels decrease rapidly after exposure stops. As all our children had stopped consuming the contaminated milk products for some time before assessment, the concentrations of melamine in both blood and urine are unhelpful. In view of the large number of children seen at our centre, a detailed history of consumption of melamine tainted milk products was not systematically recorded and was only rechecked if abnormalities found on ultrasonography were confirmed. Furthermore, as different batches of the same brand of milk product could be contaminated to varying degrees ${ }^{8}$ it was not possible to accurately calculate exposure to melamine. There was also no easy way to avoid overestimation of children's consumption of contaminated products by anxious parents. Despite these problems, dietary history and parental perceived risk of exposure are the only surrogate measures of melamine exposure available and we are therefore subject to misclassification bias.

\section{Conclusions and policy implications}

The data from our cohort suggest that large scale and urgent screening programmes may not be informative or cost effective in regions outside of the mainland of China. Evidence to show that urgent massive screening after exposure to low dose melamine will lead to any health benefits is lacking. It is possible that hyperechoic lesions at the renal papillae may be associated with exposure to low dose melamine - that is, levels below $0.63 \mathrm{mg} / \mathrm{kg} /$ day, but the clinical significance of these lesions is uncertain at this stage and long term follow-up is mandatory. In view of the lack of evidence to guide the government initially, and the large number of severely affected children on the mainland, a large scale, territory-wide urgent screening programme was probably justifiable. In light of results of our screening programme, it may now be acceptable to arrange renal assessments for select groups on routine clinical service 
to avoid stressing the already overworked public health system of Hong Kong.

The use of reagent strip testing of urine as the primary means of identifying haematuria may lead to a large number of false positive results, ${ }^{12}$ resulting in unnecessary anxiety for children and their parents. We believe, however, that reagent strip testing remains a valuable tool for identifying people for further confirmatory testing by microscopy of urine in screening programmes handling large numbers of patients in a short period. Arranging microscopy as soon as possible after a positive reagent strip result would help minimise anxiety for children and their parents.

We postulate that the difference in prevalence and severity of renal complications between our children and their counterparts on the mainland can be explained by the difference in levels of exposure to melamine. The severe acute complications observed on the mainland seem unlikely to occur elsewhere. Further medium and long term follow-up studies of these children are warranted to assess more comprehensively the public health impact of consuming milk products contaminated with melamine.

HSL and PCN contributed equally to this article. We thank our research team, So Hung Kwan, Ngai Hoi Yan, Simmy Yeung, and Jessie Mak, who helped organise and enter a large amount of data within a short period. Contributors: HSL and PCN drafted the manuscript and made substantia revisions. CKL, WW, and WCWC helped revise the manuscript. PCN, DFYC, WW, and CKL designed the screening programme at the special assessment centre. SSH, KTW, WCWC, and ATA designed the ultrasound follow-up service. WW, CKL, DFYC, KTW, ATA, SSH, and WCWC coordinated the screening service and provided the core paediatric and ultrasonographical data on which the manuscript is based. HSL is guarantor.

Funding: The government of the Hong Kong Special Administrative Region provided out of hours pay to staff working extra time during screening. The researchers were independent of the funders.

Competing interests: None declared.

Ethical approval: In light of the severe adverse renal outcomes associated with consumption of melamine tainted milk products in children on the mainland of China, an expert committee appointed by the government of the Hong Kong Special Administrative Region endorsed a screening programme comprising urinalysis, renal ultrasonography, and blood tests for renal function. There were no adverse health risks from the renal ultrasound scans and urinalysis, and only $1 \mathrm{ml}$ of whole blood was taken for plasma urea and creatinine determination. Participants were free to join the programme and therefore implied consent was assumed for the assessments and no formal written consent was required. The data presented in this report were obtained from the participants who attended our centre and are anonymised. Ethical approval for analysis and publication of the screening programme results was granted by the joint The Chinese University of Hong Kong-New Territories East Cluste Clinical Research Ethics Committee after retrospective review. Provenance and peer review: Not commissioned; externally peer reviewed.

1 Parry J. China's tainted infant formula sickens nearly 13000 babies. BMJ 2008;337:a1802.

2 Parry J. Contaminated infant formula sickens 6200 babies in China. BMJ 2008;337:a1738.

3 Chan EY, Griffiths SM, Chan CW. Public-health risks of melamine in milk products. Lancet 2008;372:1444-5.

4 World Health Organization. Melamine and cyanuric acid: toxicity, preliminary risk assessment and guidance on levels in food. Geneva: WHO, 2008. www.who.int/foodsafety/fs_management/Melamine. pdf.

5 Dobson RL, Motlagh S, Quijano M, Cambron RT, Baker TR, Pullen AM, et al. Identification and characterization of toxicity of contaminants in pet food leading to an outbreak of renal toxicity in cats and dogs. Toxicol Sci 2008;106:251-62.

6 Cianciolo RE, Bischoff K, Ebel JG, Van Winkle TJ, Goldstein RE, Serfilippi LM. Clinicopathologic, histologic, and toxicologic findings in 70 cats inadvertently exposed to pet food contaminated with melamine and cyanuric acid. J Am Vet Med Assoc 2008;233:729-37.

7 Parry J. China's tainted milk scandal spreads around world. $B M J$ 2008;337:a1890.

8 Centre for Food Safety. Frequently asked questions. 2008. www.cfs. gov.hk/english/whatsnew/whatsnew_fstr/ whatsnew_fstr_Test_dairy_product_FAQ.html.

9 Schwartz GJ, Haycock GB, Edelmann CM Jr, Spitzer A. A simple estimate of glomerular filtration rate in children derived from body length and plasma creatinine. Pediatrics 1976;58:259-63.

10 La Manna A, Polito C, Cioce F, De Maria G, Capacchione A, Rocco CE, et al. Calyceal microlithiasis in children: report on 196 cases. Pediatr Nephrol 1998;12:214-7.

11 Good LI, Edell SL, Soloway RD, Trotman BW, Mulhern C, Arger PA. Ultrasonic properties of gallstones. Effect of stone size and composition. Gastroenterology 1979;77:258-63.

12 Robbiani JH, Simonetti GD, Crosazzo L, Ferrarini A, Pronzini F, Bianchetti MG. False positive dipstick for urinary blood in childhood.J Nephrol 2006;19:605-6.

13 Pugia MJ, Lott JA, Kajima J, Saambe T, Sasaki M, Kuromoto K, et al. Screening school children for albuminuria, proteinuria and occult blood with dipsticks. Clin Chem Lab Med 1999;37:149-57.

14 Pugia MJ, Murakami M, Lott JA, Ohta Y, Kitagawa T, Yamauchi K, et al. Screening for proteinuria in Japanese schoolchildren: a new approach. Clin Chem Lab Med 2000;38:975-82.

Accepted: 8 December 2008 